\title{
Screening of some Naturally Isolated Microalgal Strains for Polyunsaturated Fatty Acids Production
}

\author{
Mohammad Hossein Morowvat ${ }^{1 *}$ and Younes Ghasemi ${ }^{1,2}$ \\ ${ }^{1}$ Pharmaceutical Sciences Research Center, School of Pharmacy, Shiraz University of Medical Sciences, \\ P.O. Box 71345-1583, Shiraz, Iran; mhmorowvat@sums.ac.ir \\ 2 Department of Pharmaceutical Biotechnology, School of Pharmacy, Shiraz University of Medical Sciences, \\ P.O. Box 71468-64685, Shiraz, Iran
}

\begin{abstract}
Background and Purpose: Nowadays, polyunsaturated fatty acids (PUFAs) are playing a great role in human wellbeing and health improvement. A wide spectrum of biological, medical and health benefit effects ranging from cardiovascular, neuronal, anticancer and antioxidant have been reported from different PUFAs in human. Methodology: In this study, six different species of microalgae belonging to the chlorophyta and cyanobacteria phylum were isolated from soil and water samples collected from Persian Gulf. Their growth rate, biomass and lipid production and productivity and more importantly their ability to produce PUFAs was investigated. Results: The isolated species represented a great fatty acid profile including many different polyunsaturated fatty acids (PUFAs) ranging from 6-20 carbon atoms. S. obliquus and $N$. muscorum proven to have a better profile for PUFAs production, whilst $C$. vulgaris could be considered as a more robust strain to produce other fatty acid classes. Besides, $C$. vulgaris with its higher growth rates $(0.39 \mathrm{~d}-1)$ and $S$. obliquus owing to its higher total lipid content (43.92\%) seems more interesting strains for scale up studies. Conclusion: The obtained results demonstrated the great potential of naturally isolated strains of microalgae for PUFA production and provided some insights in next studies to explore more producing strains.
\end{abstract}

Keywords: Lipid, Microalgae, Naturally Isolated, Polyunsaturated Fatty Acids, Screening

\section{Introduction}

Polyunsaturated fatty acids (PUFAs), are fatty acids that have more than one double bond in their chemical structure. This family of fatty acids, is comprised of some major fatty acids, such as essential fatty acids ${ }^{1}$. Omega-3, omega- 6 , and conjugated fatty acids, are considered as the main polyunsaturated fatty acids.

Docosahexaenoic acid (DHA) is a long chain PUFA from omega-3 family. DHA is known to be one of the most important constituents of the grey matter in human brain and retina. Moreover, it has been considered as a food supplement to enrich the infant formulas ${ }^{2,3}$ and dairy products such as milk and yogurt. This essential fatty acid has been investigated as a therapeutic agent for prevention and treatment in various diseases including hypertension, arthritis, arteriosclerosis, Alzheimer, depression, dementia and thrombosis ${ }^{4-6}$. Eicosapentaenoic acid (EPA) is known as another critical polyunsaturated omega-3 fatty acids which is precursor for prostaglandin, leukotriene and thromboxane biosynthesis in human. Different clinical trials have

been performed on EPA and the results warrant their effectiveness in relieving the inflammatory diseases, adjuvant therapy to cure schizophrenia or depression and also fortifying the effectiveness of chemotherapy agents ${ }^{7}$. In addition to DHA and EPA, which are classified as omega-3 fatty acids, some major fatty acids such as Alfa Linolenic Acid (ALA), Gamma Linolenic Acid (GLA) ${ }^{8}$, Arachidonic Acid (AA) ${ }^{9}$ and Eicosatrienoic Acid (ETE), which play a great role in cellular membranes in different organisms and also offer various health effects for humans,

${ }^{*}$ Author for correspondence 
have been reported from different microalgal strains ${ }^{10,11}$.

Nowadays, DHA is obtained mainly from fish oil. Nevertheless, due to shortage of resources, unpleasant odor, possible existence of heavy metals or other pollutants, low stability, variable composition and its lower quality ${ }^{12,13}$, worldwide research have been exploited to find a secure and sustainable source for PUFAs. In this context, microalgae have been considered as renewable, pollution-free and robust candidates for PUFA production ${ }^{14,15}$.

The results of previous studies indicate that some marine microalgae could be exploited as a suitable bioresource for various classes of PUFAs and especially DHA and $\mathrm{EPA}^{16}$. Owing to its diverse marine habitats such as coral reefs, sandy beaches, maritime forests, Iran have been regarded as a large and unstudied source to find unique microalgal strains with special metabolic capabilities ${ }^{17-20}$. Nevertheless, there is not a comprehensive study on naturally isolated microalgal strains from these habitats to assess the PUFA production possibility for large scale production.

Hence, in this study, during a screening program some autotrophic and heterotrophic microalgal strains were isolated and identified from water and soil samples collected from various habitats close to the Persian Gulf. The isolated microalgal strains were identified using molecular and morphologic methods. Growth rates and fatty acid profile of the six identified microalgal strains were investigated. BG11 culture medium was used for growth and preservation of microalgae. The results of this study could be of importance for identification and classification of microalgae from other habitats and also shows the pharmaceuticals and nutritional importance of these strains.

\section{Materials and Methods}

\subsection{Isolation and Identification of}

Microalgae

The studied microalgal strains in this study were isolated from regional waters and wet soil samples from different habitats located in vicinity to Persian Gulf, Bushehr, Iran. Preliminary isolation and cultivation of the microalgal strains was performed in School of Pharmacy, Shiraz University of Medical Sciences. Molecular methods such as $16 \mathrm{~S}$ and $18 \mathrm{~S}$ rRNA ribosomal genes sequencing and also morphologic studies was conducted to identify the isolated strains.

\subsection{Ribosomal Genes Extraction and Sequencing}

The genomic content of each isolated strain was extracted using heat shock method. Ribosomal genes were amplified using universal primers against $16 \mathrm{~S}$ or $18 \mathrm{~S}$ rRNA encoding genes using PCR method ${ }^{21}$. Morphologic methods as described before was conducted to confirm the results of sequencing analysis ${ }^{22}$.

\subsection{Preservation and Cultivation Conditions}

BG11 culture medium was selected for preservation and cultivation of the six isolated microalgal strains. The growth measurement experiment was performed for 28 days. The study was carried out in $500 \mathrm{~mL}$ Erlenmeyer flasks. The flasks were stored at $25^{\circ} \mathrm{C}$ in an orbital incubator shaker (PECO, Iran), agitated at $130 \mathrm{rpm}$ under the light intensity of $60 \mathrm{~mol} \mathrm{~m}^{-2} \mathrm{~s}^{-1}$.

\subsection{Microalgal Growth Measurement}

Algal biomass measured by means of dry weight. Samples $(10 \mathrm{~mL})$ of cultures centrifuged at $6000 \mathrm{~g}$, for $5 \mathrm{~min}$. Pellets were washed twice with distilled water and centrifuged again to remove impurities. The pellets dried at $88^{\circ} \mathrm{C}$ overnight to determine the biomass dry weight.

\subsection{Total Lipid Extraction and Fatty Acids Profiling}

The total lipid content of the studied strain in each experiment was extracted as described before with some modifications ${ }^{23}$. The obtained microalgal biomass $(1 \mathrm{~g})$ was boiled in isopropanol for $2 \mathrm{~min}$ to prevent the possible lipase activity. It was then homogenized in a mixture of chloroformmethanol (1:2). Moreover, butylated hydroxytoluene (BHT) $(0.05 \% \mathrm{w} / \mathrm{v})$ was added as antioxidant agent. Distilled water $(0.8 \mathrm{~mL})$, chloroform $(5 \mathrm{~mL})$ and potassium chloride aqueous solution $(0.88 \mathrm{w} / \mathrm{v})$ was

added to the mixture. It was shaken vigorously. The solvent phase was isolated and dried under nitrogen gas. It was used for total lipid determination using gravimetric method. Dien-stark apparatus was used for esterification of the isolated fatty acids. Thin layer chromatography (TLC) was performed to assess the obtained fatty acid methyl esters (FAMEs). The obtained oily substances were injected separately into GC/Mass apparatus. The GC/MS analysis was performed as described before ${ }^{24}$. 


\subsection{Statistical Analysis}

The significant differences of the obtained data $(\mathrm{n}=$ 3) was analyzed by using ANOVA with statistically difference at a level of 5\%. GraphPad prism version 6.00 (GraphPad Software, La Jolla California, USA) and IBM SPSS software version 22.0 (Armonk, NY: IBM Corp.) were used for these analysis.

\section{Results and Discussion}

\subsection{Microalgae Isolation and Identification}

According to the described molecular and morphologic methods six distinct microalgal species from different strains of chlorophyta and cyanobacteria phylum were isolated. Identification of the isolated microalgae was done using morphological studies and taxonomical approaches. The $16 \mathrm{~S}$ or $18 \mathrm{~S}$ rRNA gene sequence for each microalgal strain was amplified using previously designed universal primers. The exploited PCR protocol was according to some previously represented studies ${ }^{21,24}$. Sequencing of the amplified ribosomal genes was performed by the CinnaGen Company. The isolated microalgal strains was found to be Oocystis pusilla, Scenedesmus obliquus, Chlamydomonas reinhardtii, Chlorella vulgaris, Nostoc muscorum and Fischerella ambigua. The isolated microalgal strain were deposited in microalgal culture collection of Shiraz University of Medical Sciences (MCCS) for preservation and cultivation.

\subsection{Growth Measurements Studies}

The results of growth measurement analysis and other kinetic studies such as the maximum biomass production (Figure 1), productivity and lipid production in BG11 culture medium are shown in Table 1. Growth analysis including the maximum specific growth rate $\left(\mu_{\max }\right)$, maximum biomass concentration $\left(X_{\max }\right)$, maximum biomass productivity $\left(P_{\max }\right)$, and maximum lipid concentration $\left(\mathrm{L}_{\max }\right)$ for each

isolated strain was monitored for 28 days during cultivation. Besides, due to importance of lipid content obtained from each microalgal strain, the total percent of lipids for each strain in the final obtained biomass was also investigated (Figure 2). According to this data the minimum percent of lipids in the total obtained biomass was occurred in F. ambigua (32.14\%) and the highest amounts was found to be in S obliquus (43.92\%).

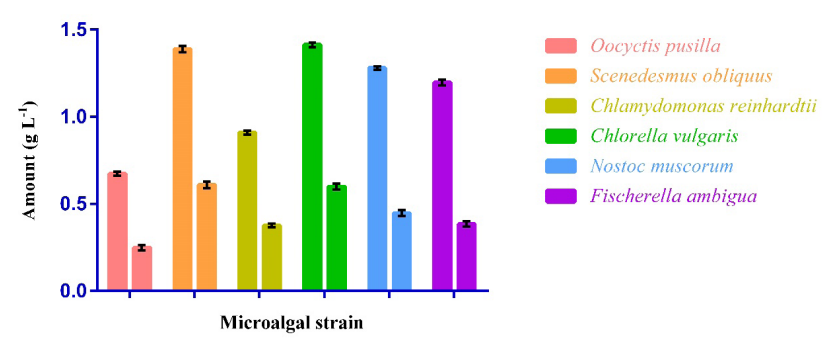

Figure 1. Total biomass production (g L-1) by each studied microalgae (left columns) and total amount of lipid ( $\mathrm{g}$ L-1) in biomass (right columns) with error bars $(\mathrm{n}=3)$.

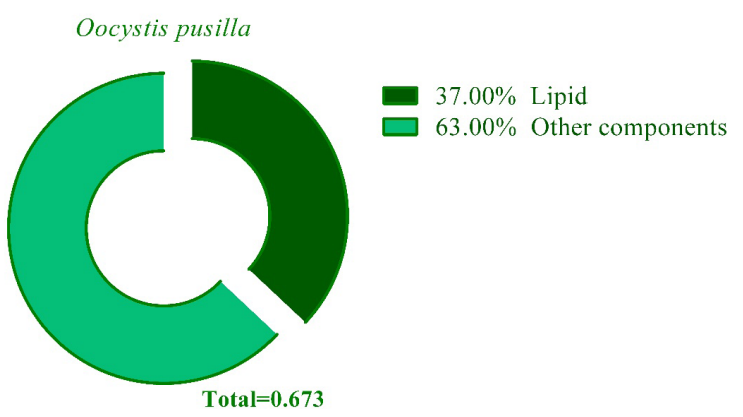

Figure 2a

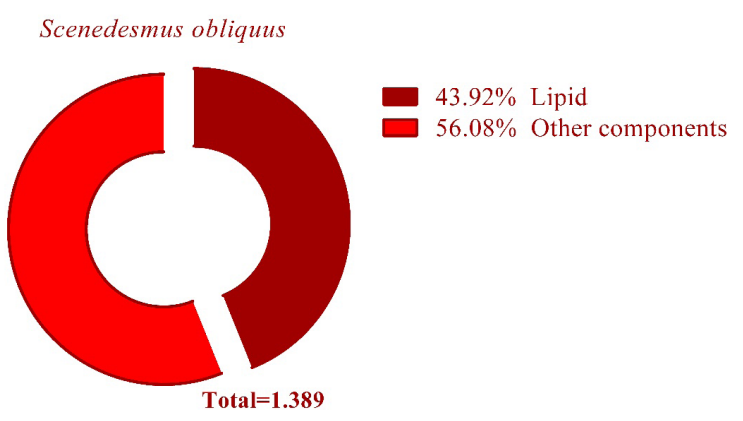

Figure 2b

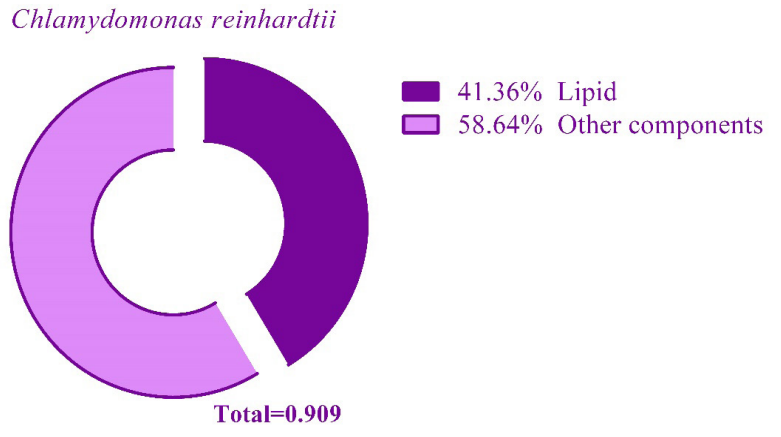

Figure 2c 


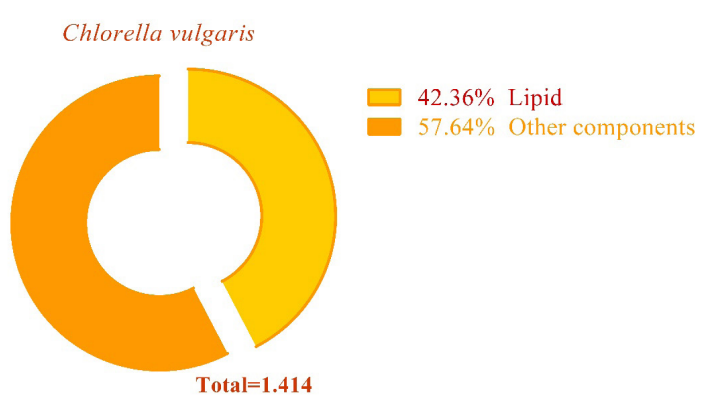

Figure 2d

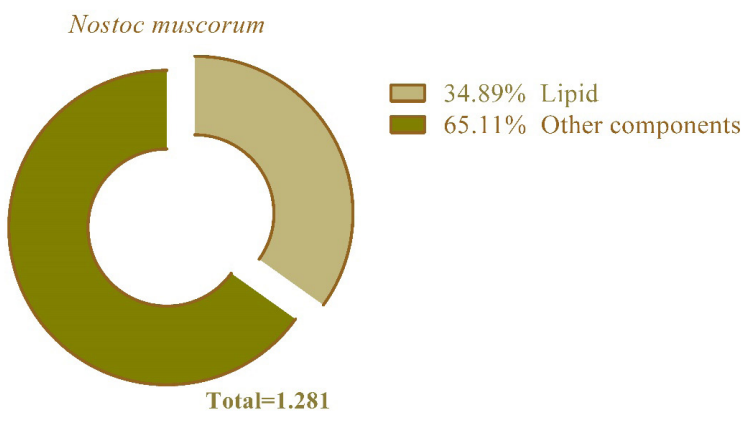

Figure 2e

Table 1. The growth parameters, maximum biomass production and productivity and lipid production in six identified microalgal strains in BG11 culture medium during 28 days of study

\begin{tabular}{lccccc}
\hline Microalgal strain & \multicolumn{2}{c}{$\begin{array}{c}\text { Growth } \\
\text { parameters }\end{array}$} & \multicolumn{2}{c}{$\begin{array}{c}\text { Biomass } \\
\text { content }\end{array}$} & $\begin{array}{c}\text { Lipid } \\
\text { content }\end{array}$ \\
\hline O. pusilla & $\mu_{\max }$ & $\mathrm{D}_{\max }$ & $\mathrm{X}_{\max }$ & $\mathrm{P}_{\max }$ & $\mathrm{L}_{\max }$ \\
S. obliquus & 0.27 & 20 & 0.673 & 0.19 & 0.249 \\
C. reinhardtii & 0.37 & 21 & 1.389 & 0.36 & 0.610 \\
C. vulgaris & 0.28 & 21 & 0.909 & 0.33 & 0.376 \\
N. muscorum & 0.39 & 23 & 1.414 & 0.37 & 0.599 \\
F. ambigua & 0.31 & 19 & 1.281 & 0.32 & 0.447 \\
& 0.33 & 20 & 1.198 & 0.32 & 0.385 \\
\hline
\end{tabular}

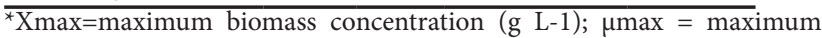
specific growth rate (d-1); Dmax= the day with maximum Xmax; Pmax $=$ maximum biomass productivity $(\mathrm{g} \mathrm{L}-1 \mathrm{~d}-1) ; \mathrm{Lmax}=$ maximum lipid concentration (g L-1)

According to the results of growth kinetic analysis, the total obtained biomass $\left(X_{\max }\right)$ varied from the minimum of $0.673 \mathrm{~g} \mathrm{~L}^{-1}$ in O. pusilla to the maximum of $1.414 \mathrm{~g}$ $\mathrm{L}^{-1}$ for C. vulgaris. The day with the maximum biomass $\left(D_{\max }\right)$ was between 19-23 days after commencing the cultivation. The maximum specific growth rates $\left(\mu_{\max }\right)$ differed between $0.27 \mathrm{~d}^{-1}$ for $O$. pusilla to $0.39 \mathrm{~d}^{-1}$ in $C$. vulgaris strain. When compared with the reported growth kinetics from other microalgal strains ${ }^{25}$, it could be concluded that the obtained $X_{\max }$ and $\mu_{\max }$ for our study seems quite acceptable for a potentially producing microalgal strain. The maximum biomass productivity was found in C. vulgaris with $0.37 \mathrm{~g} \mathrm{~L}^{-1} \mathrm{~d}^{-1}$. On the other hand, the minimum biomass productivity was reported in O. pusilla with just $0.19 \mathrm{~g} \mathrm{~L}^{-1} \mathrm{~d}^{-1}$. The results of lipid production analysis comprehend a maximum lipid concentration $\left(\mathrm{L}_{\max }\right)$ between $0.249-610 \mathrm{~g} \mathrm{~L}^{-1}$ for O. pusilla and $S$. obliquus respectively. It could be concluded that $C$. vulgaris with

its higher growth rates and S. obliquus owing to its higher total lipid content seems more interesting strains for cultivation and aquaculture studies for biomass and lipid production. On the other hand, O. pusilla showed the least potential for such applications.

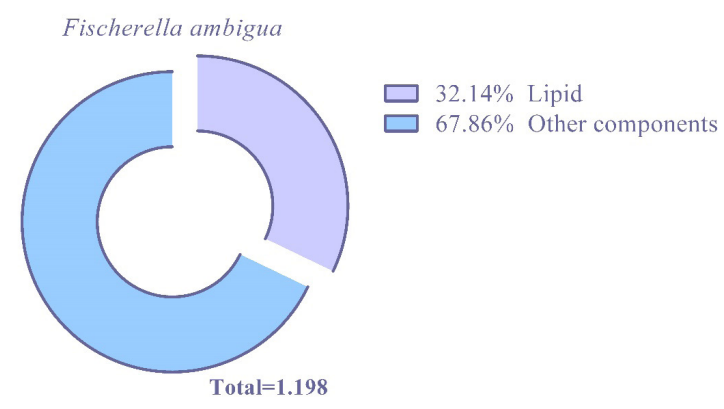

Figure 2a-2f. The total lipid content of each identified microalgal strain $(2 \mathrm{a}-2 \mathrm{f})(\% \mathrm{w} / \mathrm{w})$ in the final obtained biomass.

\subsection{Fatty Acid Analysis}

After entering to the stationary phase of microalgal growth, total lipids in the obtained biomass from six strains of microalgae were primarily esterified and then identified through TLC and GC/MS analysis. Progression of methyl esterification procedure on the extracted fatty acids was observed using TLC method accompanied by spraying solution of vanillin-sulfuric acid. The retention factor $\left(\mathrm{R}_{\mathrm{f}}\right)$ values of TLC analysis are given in Table 2 (solvent system: chloroform). The GC/MS analyses were carried out on the product of esterification (FAs/FAMEs) in the above mentioned microalgae strains. 
Table 2. The retention time (Rf) values in TLC analysis for each FAME from the six species of naturally isolated microalgae (solvent system: chloroform)

\begin{tabular}{lc}
\hline Microalgal strain & $\mathbf{R}_{\mathrm{f}}$ for FAMEs \\
\hline Oocystis pusilla & 0.68 \\
Scenedesmus obliquus & 0.72 \\
Chlamydomonas reinhardtii & 0.80 \\
Chlorella vulgaris & 0.69 \\
Nostoc muscorum & 0.76 \\
Fischerella ambigua & 0.80 \\
\hline
\end{tabular}

The identification of FAs/FAMEs was performed through the comparison of their mass spectra with those in Wiley libraries ${ }^{26}$. The results of such analysis were classified as a profile composed of the systematic name for each identified fatty acid (FA)/fatty acid methyl ester (FAME), number of carbon atoms in the structure of FA/FAME, number of occurred double bond(s) and its position in the molecule with fatty acid's family according to saturation/unsaturation in Tables 3. As it has been shown different types of FAs/FAMEs ranging from 3-38 carbon atoms were detected in the six studied naturally isolated microalgae. The smallest fatty acid was found to be 2-Propenoic acid which occurred in O. pusilla and N. muscorum. Whilst octatriacontanoic acid with 38 carbon atoms was the largest fatty acid which identified in C. vulgaris. Some FAs/FAMEs were found to be more unique which only founded in a certain strain. To be more precise, 5-Hexenoic acid (O. pusilla); 2-Heptenoic acid, eicosenoic acid, triacontanoic acid (S. obliquus); nonanoic acid, decanoic acid, methyl decanoate, 16-Octadecanoic acid (C. reinhardtii); 9-Hexadecenoic acid, methyl hexadecanoate, 15-Octadecenoic acid (C. vulgaris); eicosanoic acid (N. muscorum); 2-Pentenoic acid (F. ambigua) was only found in mentioned strain. In this context, could be considered as a metabolic selection marker for future studies or other researchers. In contrast, 6-Octadecenoic acid was found in all six analyzed microalgae. So this fatty acid could not be used as a selective marker for this strains of microalgae. Lipid profiling ${ }^{27,28}$ the results of this analysis might be of help for classification of the other microalgal strains living in this ecosystem and also for the identification of new strains.

The results of fatty acid profiling study also revealed that some monounsaturated fatty acids (MUFA) such as 2-Propenoic acid (O. pusilla and N. muscorum), 2-Hexenoic acid (C. reinhardtii and F. ambigua),
3-Hexenoic acid (C. reinhardtii, C. vulgaris and $N$. muscorum), 5-Hexenoic acid (O.pusilla), 2-Heptenoic acid (S. obliquus), 3-Octenoic acid (S. obliquus, C. vulgaris and F. ambigua), 2-Pentenoic acid (F. ambigua), 4-Pentenoic acid (O. pusilla, S. obliquus, C. vulgaris, and N. muscorum), 7-Hexadecenoic acid (S. obliquus, and C. vulgaris), 9-Hexadecenoic acid (C. vulgaris), 6-Octadecenoic acid (O. pusilla, S. obliquus, C. reinhardtii, C. vulgaris, $N$. muscorum, and F. ambigua), 8-Octadecenoic acid (O. pusilla, C. reinhardtii, and F. ambigua), 9-Octadecenoic acid (O. pusilla, and C. vulgaris), 10-Octadecenoic acid (S. obliquus and N. muscorum), 15-Octadecenoic acid (C. vulgaris), 16-Octadecenoic acid (C. reinhardtii), eicosenoic acid (S. obliquus) and 15-Tetracosenoic acid (C. vulgaris and F. Ambigua); exists in the extracted lipids.

The major PUFAs with two double bonds were found to be 2, 4-Hexadienedioic acid (S. obliquus, C. reinhardtii, N. muscorum, and F. ambigua); 9,12-Hexadecadienoic acid (S. obliquus, and N. muscorum); 9,12-Octadecadienoic acid (S. obliquus, C. vulgaris, and F. ambigua); 9,15-Octadecadienoic acid (C. reinhardtii, N. muscorum, and F. ambigua); 10,13-Octadecadienoic acid (C. vulgaris, and N. muscorum); 12,15-Octadecadienoic acid (S. obliquus and N. muscorum); and 9,11-Octadecadienoic acid (O. pusilla, S. obliquus, C. reinhardtii and F. ambigua).

Moreover, PUFAs with three double bonds were identifies as 7,10,13-Hexadecatrienoic acid (O. pusilla, $S$. obliquus and F. ambigua); 9,12,15-Octadecatrienoic acid ( $O$. pusilla, S. obliquus and C. vulgaris); 6,9,12-Octadecatrienoic acid (O. pusilla, C. reinhardtii, C. vulgaris and N. muscorum); and 11,14,17-Eicosatrienoic acid (O. pusilla, C. reinhardtii and N. muscorum) (Figure 3).

As the results indicate our isolated strains did not exhibit the ability to produce some valuable PUFAs such as DHA or EPA. Nevertheless, regarding the noticeable growth rates and lipid productivity and a good profile for other PUFAs, the studied strains might be considered as potential candidates for scale up studies. The major omega-3 fatty acids comprehend to be 7,10,13-Hexadecatrienoic acid $(n-3,6,9)$, 9,15-Octadecadienoicacid (n-3,9),12,15-Octadecadienoic acid $(n-3,6), 9,12,15$-Octadecatrienoic acid $(n-3,6,9)$ and 11,14,17-Eicosatrienoic acid $(n-3,6,9)$.

The total number of identified FAs/FAMEs in six studied strain was ranging from 20 variants (O. pusilla) to 30 (C. vulgaris) different types. Containing seven distinct PUFAs, S. obliquus and N. muscorum show more 
fascinating FA/FAME profile for further studies. On the other hand, C. vulgaris with only four different types of PUFAs showed the lowest variants of PUFAs (Figure 4).

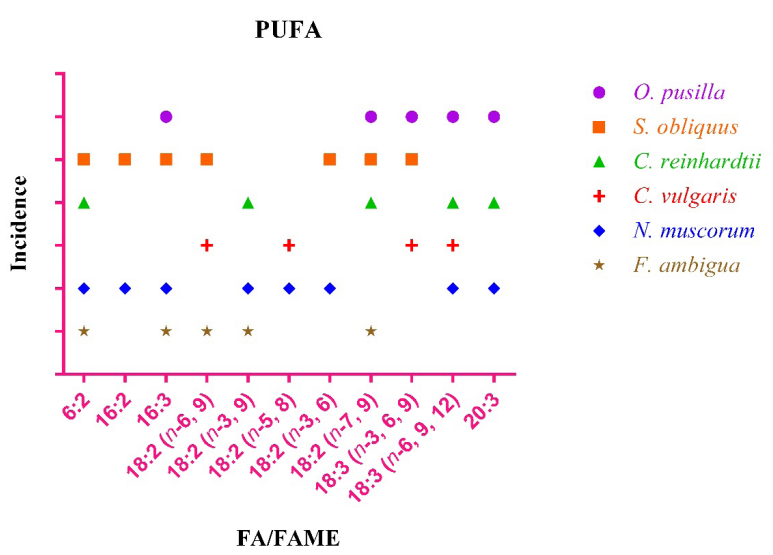

Figure 3. Total polyunsaturated fatty acids obtained from each identified microalgal strain ranging from 6-20 carbon atoms.

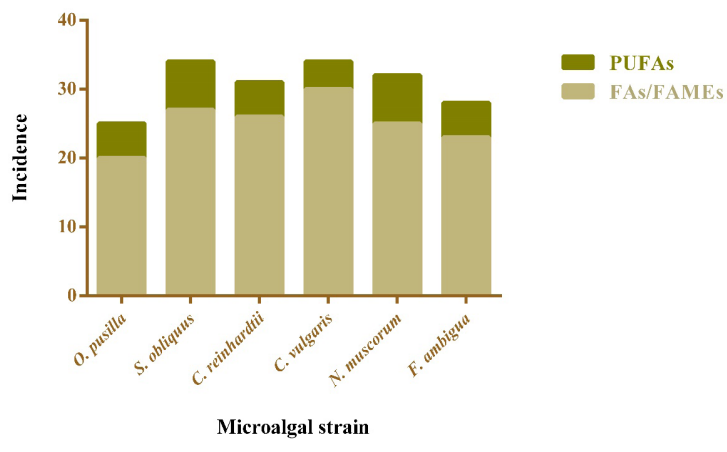

Figure 4. Total number of identified fatty acids/fatty acid methyl esters (the whole column) from each studied strain and their polyunsaturated fatty acid variants (the upper segment of each column).

Based upon the results of this study, S. obliquus and N. muscorum show more promise for different PUFAs production and $C$. vulgaris revealed to be the more producer for other classes of fatty acids.

Table 3. List of identified fatty acids (FAs)/fatty acid methyl esters (FAMEs) in the six isolated microalgae with their carbon atoms and number of double bonds

\begin{tabular}{|c|c|c|c|c|c|c|c|}
\hline FA/FAME & $\begin{array}{c}O . \\
\text { pusilla }\end{array}$ & $\begin{array}{c}\text { S. } \\
\text { obliquus }\end{array}$ & $\begin{array}{c}C . \\
\text { reinhardtii }\end{array}$ & $\begin{array}{c}C . \\
\text { vulgaris } \\
\end{array}$ & $\begin{array}{c}N . \\
\text { muscorum }\end{array}$ & $\begin{array}{c}F . \\
\text { ambigua }\end{array}$ & $\begin{array}{c}\text { No. of carbons: No. of } \\
\text { double bond(s) }\end{array}$ \\
\hline 2-Propenoic acid & + & & & & + & & $3: 1$ \\
\hline Butanoic acid & & + & & + & & & 4:0 \\
\hline 2-Methyl-2-propenoic acid & & & + & + & & + & 4:0 \\
\hline Pentanoic acid & + & + & & & & + & 5:0 \\
\hline Hexanoic acid & & & + & + & & & $6: 0$ \\
\hline 2-Hexenoic acid & & & + & & & + & $6: 1$ \\
\hline 3-Hexenoic acid & & & + & + & + & & $6: 1$ \\
\hline 5-Hexenoic acid & + & & & & & & $6: 1$ \\
\hline 2, 4-Hexadienedioic acid & & + & + & & + & + & $6: 2$ \\
\hline Heptanedioic acid & & & & + & & + & $7: 0$ \\
\hline 2-Heptenoic acid & & + & & & & & $7: 1$ \\
\hline Octanoic acid & & + & & + & + & & $8: 0$ \\
\hline 3-Octenoic acid & & + & & + & & + & $8: 1$ \\
\hline Nonanoic acid & & & + & & & & 9:0 \\
\hline Decanoic acid & & & + & & & & 10:0 \\
\hline Undecanoic acid & + & + & & + & + & + & 11:0 \\
\hline Methyl decanoate & & & + & & & & 11:0 \\
\hline Dodecanoic acid & & + & & & + & & 12:0 \\
\hline Methyl undecanoate & + & & & & + & + & 12:0 \\
\hline Tridecanoic acid & & & + & + & & & 13:0 \\
\hline Methyl dodecanoate & & + & + & & & + & 13:0 \\
\hline Tetradecanoic acid & & + & + & + & + & & 14:0 \\
\hline
\end{tabular}


Pentadecanoic acid

Methyl tetradecanoate

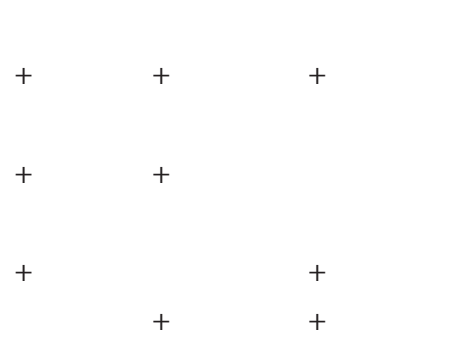

$+$

$\begin{array}{cccc} & + & 15: 0 \\ + & & 15: 0 \\ & + & 15: 1 \\ + & & 15: 1 \\ + & & 16: 0 \\ + & + & 16: 0 \\ & & 16: 1 \\ & & 16: 1\end{array}$

9-Hexadecenoic acid

9,12-Hexadecadienoic acid

7,10,13-Hexadecatrienoic acid

$+$

Heptadecanoic acid
Methyl hexadecanoate

Octadecanoic acid

Methyl heptadecanoate

6-Octadecenoic acid

8-Octadecenoic acid

9-Octadecenoic acid

10-Octadecenoic acid

15-Octadecenoic acid

9,15-Octadecadienoic acid

10,13-Octadecadienoic acid

12,15-Octadecadienoic acid

9,11-Octadecadiynoic acid

9,12,15-Octadecatrienoic acid

6,9,12-Octadecatrienoic acid

Nonadecanoic acid

Eicosanoic acid

Eicosenoic acid

11,14,17-Eicosatrienoic acid

Heneicosanoic acid

Docosanoic acid

Tricosanoic acid

Tetracosanoic acid

15-Tetracosenoic acid

Heptacosanoic acid

Triacontanoic acid

Octatriacontanoic acid
16-Octadecanoic acid

9,12-Octadecadienoic acid

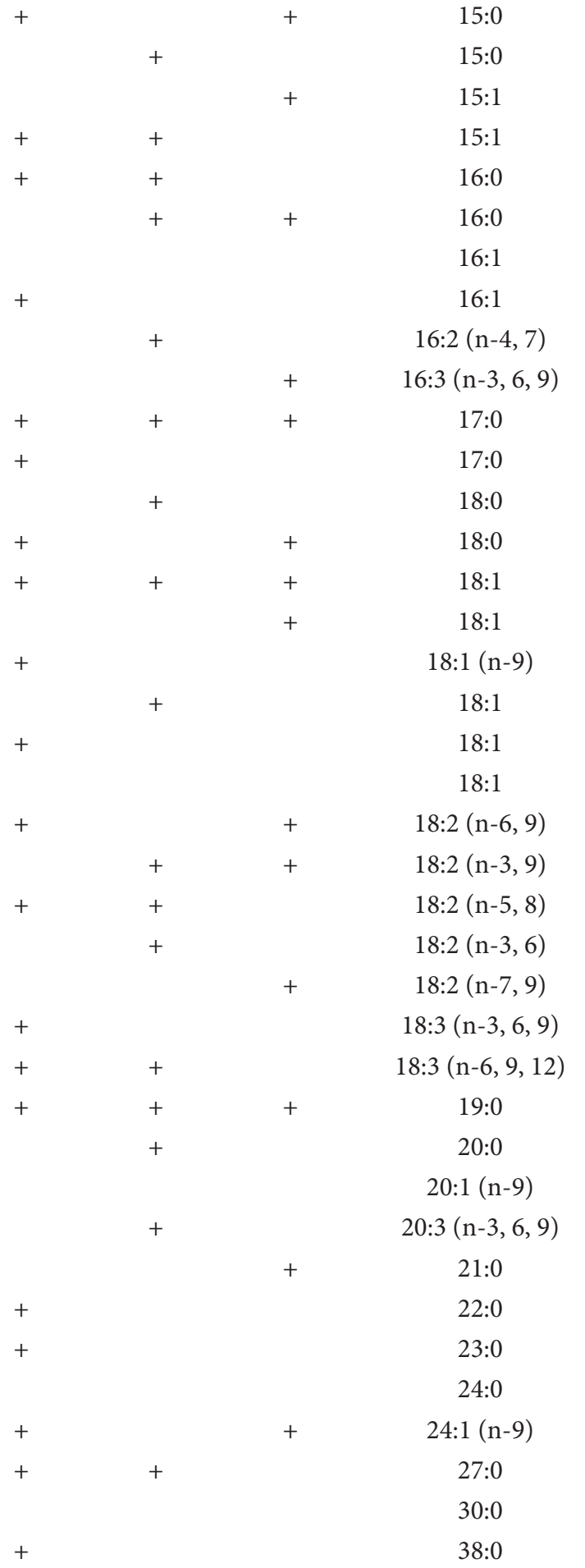

Recently some newly emerged technologies such as metabolic engineering has been successfully exploited to increase the omega-3 production yield up to the highest known amounts in dry cell weight biomass in Yarrowia lipolytica yeast ${ }^{29,30}$. With increasing information on different microalgal genes and genomes, it could be suggested that genetic, metabolic and bioprocess engineering approach seem rational to maximize the microalgal production platform for PUFAs production. Finding sheds light on defined avenue in this study towards finding a sustainable and non-animal resource for PUFAs. 


\section{Conclusion}

To sum up, according to growth kinetics and fatty acids profiles, S. obliquus and N. muscorum proven to have a better profile for PUFAs production, whilst C. vulgaris could be considered as a more robust strain to produce other fatty acid classes. The results of this study indicate that changing towards PUFA production from microalgae offers a crucial revolution to solve the increasing demands for this category of lipids. Present microalgal production plants for PUFA and other value-added food is still in infancy but due to its environmental advantages and commercial concerns and also its scalability it seems being developed in future. Moreover, only a few part of the microalgal strains in the world have been studied for their composition and potential for large scale production of metabolites and biomass. Hence, it seems essential to investigate other habitats to find the more robust microalgal strains.

\section{Acknowledgements}

This work was supported by Research Deputy of Shiraz University of Medical Sciences, Shiraz, Iran (Grant no. 93-01-36-7750).

\section{References}

1. Gill I, Valivety R. Polyunsaturated fatty acids, part 1: Occurrence, biological activities and applications. Trends in Biotechnology. 1997; 15(10):401-9.

2. Auestad N, Scott DT, Janowsky JS, Jacobsen C, Carroll RE, Montalto MB, et al. Visual, cognitive, and language assessments at 39 months: a follow-up study of children fed formulas containing long-chain polyunsaturated fatty acids to 1 year of age. Pediatrics. 2003; 112(3 Pt 1):e177-83.

3. Fleith M, Clandinin MT. Dietary PUFA for preterm and term infants: review of clinical studies. Critical Reviews in Food Science and Nutrition. 2005; 45(3):205-29.

4. Alessandri JM, Guesnet P, Vancassel S, Astorg P, Denis I, Langelier B, et al. Polyunsaturated fatty acids in the central nervous system: evolution of concepts and nutritional implications throughout life. Reproduction, Nutrition, Development. 2004; 44(6):509-38.

5. Brenna JT, Diau GY. The influence of dietary docosahexaenoic acid and arachidonic acid on central nervous system polyunsaturated fatty acid composition. Prostaglandins, Leukotrienes, and Essential Fatty Acids. 2007; 77(5-6):247-50.

6. Das UN. Folic acid and polyunsaturated fatty acids improve cognitive function and prevent depression, dementia, and
Alzheimer's disease-but how and why? Prostaglandins, Leukotrienes, and Essential Fatty Acids. 2008; 78(1):11-9.

7. Mullen A, Loscher CE, Roche HM. Anti-inflammatory effects of EPA and DHA are dependent upon time and dose-response elements associated with LPS stimulation in THP-1-derived macrophages. The Journal of Nutritional Biochemistry. 2010; 21(5):444-50.

8. Hirano M, Mori H, Miura Y, Matsunaga N, Nakamura $\mathrm{N}$, Matsunaga T. $\gamma$-linolenic acid production by microalgae. Applied Biochemistry and Biotechnology. 1990; 2425(1):183-91.

9. Nichols BW, Appleby RS. The distribution and biosynthesis of arachidonic acid in algae. Phytochemistry. 1969; 8(10):1907-15.

10. Guedes AC, Amaro HM, Barbosa CR, Pereira RD, Malcata FX. Fatty acid composition of several wild microalgae and cyanobacteria, with a focus on eicosapentaenoic, docosahexaenoic and $\alpha$-linolenic acids for eventual dietary uses. Food Research International. 2011; 44(9):2721-9.

11. Ghasemi Y, Rasoul-Amini S, Morowvat MH. Algae for the production of SCP. In: Liong MT, editor. Bioprocess Sciences and Technology: Nova Science Publishers, Inc. 2011. p. 163-84.

12. De Swaaf ME, Sijtsma L, Pronk JT. High-cell-density fedbatch cultivation of the docosahexaenoic acid producing marine alga Crypthecodinium cohnii. Biotechnology and Bioengineering. 2003; 81(6):666-72.

13. Guil-Guerrero JL, Belarbi EIH, Rebolloso-Fuentes MM. Eicosapentaenoic and arachidonic acids purification from the red microalga Porphyridium cruentum. Bioseparation. 2000; 9(5):299-306.

14. Spolaore P, Joannis-Cassan C, Duran E, Isambert A. Commercial applications of microalgae. Journal of Bioscience and Bioengineering. 2006; 101(2):87-96.

15. Tonon T, Harvey D, Larson TR, Graham IA. Long chain polyunsaturated fatty acid production and partitioning to triacylglycerols in four microalgae. Phytochemistry. 2002; 61(1):15-24.

16. Khozin-Goldberg I, Iskandarov U, Cohen Z. LC-PUFA from photosynthetic microalgae: Occurrence, biosynthesis, and prospects in biotechnology. Applied Microbiology and Biotechnology. 2011; 91(4):905-15.

17. Ghasemi Y, Faramarzi MA, Arjmand-Inalou M, Mohagheghzadeh A, Shokravi S, Morowvat MH. Side-chain cleavage and C-20 ketone reduction of hydrocortisone by a natural isolate of Chroococcus dispersus. Annals of Microbiology. 2007; 57(4):577-81.

18. Ghasemi Y, Rasoul-Amini S, Morowvat MH, Raee MJ, Ghoshoon MB, Nouri F, et al. Characterization of hydrocortisone biometabolites and 18S rRNA gene in Chlamydomonas reinhardtii cultures. Molecules. 2008;13(10):2416-25.

19. Shaker S, Nemati A, Montazeri-Najafabady N, Mobasher MA, Morowvat MH, Ghasemi Y. Treating urban wastewater: Nutrient removal by using immobilized green algae in batch cultures. International Journal of Phytoremediation. 2015; 17(12):1177-82. 
20. Ghasemi Y, Mohagheghzadeh A, Moshavash M, Ostovan Z, Rasoul-Amini S, Morowvat MH, et al. Biotransformation of monoterpenes by Oocystis pusilla. World Journal of Microbiology and Biotechnology. 2009; 25(7):1301-4.

21. Ghasemi Y, Rasoul-Amini S, Kazemi A, Zarrinic G, Morowvat $\mathrm{MH}$, Kargar $\mathrm{M}$. Isolation and characterization of some moderately halophilic bacteria with lipase activity. Mikrobiologiia. 2011; 80(4):477-81.

22. Ghasemi Y, Rasoul-Amini S, Morowvat MH, Azam SBM, Shokravi S, Mohagheghzadeh A, et al. Bioconversion of hydrocortisone by unicellular microalga Oocystis pusilla. Biotechnology. 2008; 7(2):293-8.

23. Morowvat MH, Rasoul-Amini S, Ghasemi Y. Chlamydomonas as a "new" organism for biodiesel production. Bioresource Technology. 2010; 101(6):2059-62.

24. Rasoul-Amini S, Ghasemi Y, Morowvat MH, Mohagheghzadeh A. PCR amplification of $18 \mathrm{~S}$ rRNA, single cell protein production and fatty acid evaluation of some naturally isolated microalgae. Food Chemistry. 2009; 116(1):129-36.

25. Lee E, Jalalizadeh M, Zhang Q. Growth kinetic models for microalgae cultivation: A review. Algal Research. 2015;12:497-512.
26. Ghasemi Y, Khalaj A, Mohagheghzadeh A, Khosravi AR, Morowvat MH. Composition and antimicrobial activity of the essential oil and extract of Hypericum elongatum. Journal of Applied Sciences. 2007; 7(18):2671-5.

27. Lang I, Hodac L, Friedl T, Feussner I. Fatty acid profiles and their distribution patterns in microalgae: A comprehensive analysis of more than 2000 strains from the SAG culture collection. BMC Plant Biology. 2011; 11(1):1-16.

28. Griffiths MJ, Harrison STL. Lipid productivity as a key characteristic for choosing algal species for biodiesel production. Journal of Applied Phycology. 2009; 21(5):493-507.

29. Xue Z, Sharpe PL, Hong S-P, Yadav NS, Xie D, Short DR, et al. Production of omega-3 eicosapentaenoic acid by metabolic engineering of Yarrowia lipolytica. Nature Biotechnology. 2013; 31(8):734-40.

30. Xie D, Jackson EN, Zhu Q. Sustainable source of omega-3 eicosapentaenoic acid from metabolically engineered Yarrowia lipolytica: from fundamental research to commercial production. Applied Microbiology and Biotechnology. 2015; 99:1599-610. 